\title{
DETERMINATION OF SEWING THREAD CONSUMPTION FOR 602, 605, AND 607 COVER STITCHES USING GEOMETRICAL AND MULTI-LINEAR REGRESSION MODELS
}

\author{
Malek Sarah¹, Jaouachi Boubaker ${ }^{1}$, Adolphe Charles Dominique $^{2}$ \\ 1 Textile Engineering Laboratory, National School of Engineers of Monastir, University of Monastir, Monastir, Tunisia \\ 2Laboratoire de Physique et Mécanique Textile, Ecole Nationale Supérieure d'Ingénieurs Sud Alsace, Université de Haut-Alsace, France \\ ${ }^{*}$ Corresponding author. E-mail: maleksarah88@gmail.com
}

\begin{abstract}
:
This work deals with prediction of the quantities of sewing threads required to sew a garment using cover stitches for the different classes of 600 (602, 605 and 607 cover stitches) by performing a rapid and precise methods (Geometrical and statistical). Sewing consumption value was estimated based on the geometrical method of different cover stitch shapes to prevent inventory of stocks for each stitch type. In the prediction of the sewing thread consumption for each investigated stitch, it was assumed that the consumption is a function of the input parameters, such as material thickness, stitch density, yarn linear density, and seam width. In addition, a statistical method based on multi-linear regression was established. The coefficient $R^{2}$ value was determined to evaluate the precision of the geometrical and statistical methods. By comparing the theoretical thread consumption with the experimental ones, it is concluded that the geometrical method is more accurate than the statistical method regarding the range of $R^{2}$ (from $98.78 \%$ to $99.38 \%$ ), which encouraged industrialists to use geometrical models to predict thread consumption.
\end{abstract}

The input parameters contributing to the sewing thread consumption behavior were studied and analyzed. It was concluded that the most important parameters influencing thread consumption are stitch density followed by seam width. Both yarn density and material thickness have a low contribution to the thread consumption value.

\section{Keywords:}

Consumption; apparel industry; cover stitches of the class 600; geometrical and multi-linear regression models; input parameters

\section{Abbreviations}

$n_{1}, n_{2}, n_{3}$, Needle threads (needle 1,2 , and 3 respectively); ul, Upper looper thread; Il, Lower looper thread; MT, Material thickness; $n$, Stitch density expressed by stitch number/cm; $L$, Sewing length; $S L$, Stitch length; $d$, Sewing thread diameter; Tex, Yarn linear density; $B_{1}, B_{2}, B_{3}$, Distance between two needles expressed in $\mathrm{cm}$ (needle 1 and 2, 2 and 3, 3 and 4, respectively); $C_{602}$, Thread consumption per centimeter of cover stitch type 602; $C_{605}$, Thread consumption per centimeter of cover stitch type 605; $C_{607}$, Thread consumption per centimeter of cover stitch type $607 ; C_{\mathrm{Th}}$, Theoretical value of thread consumption; $C_{\text {Exp }}$, Experimental value of thread consumption; $\hat{v}_{i}$, Vector of the expected values of the response; $v_{i}$, Vector of the observed values of the response; $\bar{v}$, Average value of the answer.

\section{Introduction}

Many components and various processes are required as well as multiple parameters must be considered to produce a garment, such as the fabric, the seam thread, machinery, and human skills. However, the consumption of fabric and seam thread remains very important among the parameters and so those parameters are analyzed. In fact, the fabric and sewing thread consumption must be analyzed to predict the adequate consumption to get rid of unnecessary inventory stocks of threads so that the operational expenses are effectively reduced and investment in stocks has considerably reduced. The sewing thread consumption has been a complicated problem for many years and scientists have tried to give industries effective methods to help the manufacturers to estimate their consumed thread based on stitch types prior to starting seaming process. The sewing thread consumption depends upon several parameters, hypotheses, and assumptions, and so the researchers used different techniques (statistical, mathematical, etc.) for estimation. But still industrialists are searching for reliable and efficient solutions.

According to literature, many studies have analyzed woven and knitted garment problems to find an appropriate method to evaluating the amount of sewing thread required to prepare garments ([1-3]). However, the determination of the exact consumption value is still a daunting task. In fact, the industrial consumptions of sewing thread depend on different parameters, such as yarn count, fabric mass, wastage, rate of breaks during sewing, stitch density, etc., which makes it difficult to identify the appropriate method for estimating threads amount. Presumptions and approximations are made especially when 
seam type, stitch density, and material thickness are the main analyzed input parameters. Indeed, these variables change according to the presumed garment's type. However, a few investigations have been done to determine the relationship between the sewing machine parameters, the thread insertion inputs, the clothing morphology factors simultaneously and the consumed thread [2]. But, in any case, the thread consumption value cannot be considered as a standard one for sewn garment (such as jean pants, shirts, and jackets). Lauriol has estimated waste percentage value of sewing thread by 10 to $15 \%[4]$.

So far, the study for efficient method is not over and has been continuing until now. Recently, Midha has found sewing thread consumption for lockstitch based on regression model [5]. Abher and khedher considered the geometrical model to calculate the consumption of the sewing thread for a 301 lock stitch $[2,6]$. But, Jaouachi provided an accurate method to predict the amount of sewing thread required to stitch woven fabric using lockstitch (301) and chain stitch (401) using different methods, which are regression models, neural regression, geometrical model and fuzzy method [1, 7, 8]. Sharma and Vlinay calculated the sewing thread consumption for chain stitch and lockstitch, respectively, using regression model $[9,10]$. Abher has used a geometrical model to determine the consumption of sewing thread for 504 over-edge stitch [11]. Furthermore, Gazzah et al. has developed a model based on 602 geometrical cover-stitches to calculate the relative consumption of sewing thread length [12]. Vaseem et al. estimated thread consumption by determining an elliptical model for lockstitch 301seam [13]. Jaouachi et al [14] compared basic stitch's consumptions using image analysis, geometrical modeling, and statistical techniques. Also, Sarah et al. determined the sewing thread consumption for different lockstitches of Class 300, chain stitches Class of 400 and overedge stitches of Class 500 using geometrical and multi-linear regression models [15-17].

Although the prediction of thread consumption has been studied for the $301,401,504,516$ and 602 stitches in the literature, only the consumption of point 602 wire for class 600 has been studied (there is almost a few works dealing with the determination of the seam thread consumption of 602 , for example Gazzah study) [12]. Moreover, there is no work related to the determination of the seam thread consumption of different type of stitches in the same class.

In this study, we worked on different cover stitches of class 600 , which are used in industries for sew clothing (namely denim garments, hems of knitwear, for the laying of mesh fabric, and lace underwear and lingerie).

This work aims to accurately determine the amount of sewing thread required to stitch a specific length of woven fabric using three cover stitch types of class 600 (602, 605 and 607 cover stitches). In fact, two different methods namely geometrical method and a multi-linear regression method are proposed and compared to select the best one for the prediction of thread consumption to help industrialists evaluate accurately the consumption amount of sewing threads. These two methods are selected considering the factors that they are the simplest, easy to be used by industrialists, and are easily implemented. They do not need special software to determine the sewing thread consumption; they get the result by using a simple equation in case it is effectively proved. Further, the effect of each input parameter has been studied and discussed to determine the most significant factors to regulate before launching production.

\section{Material and methods}

\subsection{Fabric properties}

Six commercial denim fabrics were chosen for our study. They have different characteristics namely thicknesses and blend compositions (chosen to cover a wide range of thicknesses and compositions). These samples and their properties are

Table 1. Fabric properties

\begin{tabular}{|c|c|c|c|c|c|c|c|c|c|c|c|}
\hline Parameter & $\begin{array}{l}\text { Warp } \\
\text { yarn }\end{array}$ & $\begin{array}{l}\text { Weft } \\
\text { yarn }\end{array}$ & \multicolumn{2}{|c|}{$\begin{array}{l}\text { Linear density } \\
\text { (tex) }\end{array}$} & \multicolumn{2}{|c|}{ Mass per $\mathrm{m}^{2}$} & \multicolumn{2}{|c|}{ Thickness } & \multicolumn{3}{|c|}{ Tensile properties } \\
\hline $\begin{array}{c}\text { Fabric } \\
\text { composition } \\
\mathbf{n}^{\circ}\end{array}$ & & & Warp & Weft & $\begin{array}{l}\text { Mass } \\
\left(\mathrm{g} / \mathrm{m}^{2}\right)\end{array}$ & $\begin{array}{l}\text { CV } \\
(\%)\end{array}$ & $\begin{array}{l}\text { Thickness } \\
\text { (mm) }\end{array}$ & $\begin{array}{l}\text { CV } \\
(\%)\end{array}$ & $\begin{array}{l}\text { Breaking } \\
\text { force }(\mathrm{N})\end{array}$ & $\begin{array}{c}\text { Elongation } \\
\text { at break } \\
(\%)\end{array}$ & $\begin{array}{l}\text { CV } \\
(\%)\end{array}$ \\
\hline \multirow{2}{*}{1} & 26 & 17 & 11.9 & 20 & 323 & 1.9 & 1.2 & 1.4 & 413.63 & 12.66 & 1.4 \\
\hline & 30 & 22 & 12.5 & 16.67 & 387 & 2.1 & 1.04 & 1 & 1071.90 & 17.89 & 2.1 \\
\hline \multirow{2}{*}{2} & 32 & 17 & 11.36 & 14.92 & 445 & 1.5 & 0.96 & 1.4 & 894.26 & 17.40 & 0.8 \\
\hline & 29 & 22 & 12.5 & 27.78 & 342 & 1.2 & 0.90 & 1.6 & 624.21 & 30.45 & 1.2 \\
\hline \multirow{2}{*}{3} & 31 & 21 & 12.5 & 27.78 & 334 & 1.6 & 0.85 & 1.2 & 557.24 & 33.64 & 0.6 \\
\hline & 28 & 22 & 14.29 & 27.78 & 328 & 0.9 & 0.75 & 2.1 & 528.13 & 25.12 & 2.4 \\
\hline
\end{tabular}


presented in Table 1. Each test is repeated five times for each sample according to French standards.

With:

- Fabric composition no. 1: 100\% cotton warp and weft threads;

- Fabric composition no. 2: $100 \%$ cotton warp yarn and weft yarn in $95 \%$ cotton and $5 \%$ elastane;

- Fabric Composition no. 3: 100\% cotton warp yarn and weft yarn in $71 \%$ cotton, $5 \%$ elastane, and $24 \%$ polyester.

The studied denim fabrics were fabricated using a SULZER P7300 weaving loom projectile with $3 / 1$ twill structure. This technology was used by the largest Tunisian denim fabric manufacturing industry where denim fabrics are generally made of cotton, cotton/elastane or cotton/polyester/elastane. These specific compositions are considered in this study.

\subsection{Sewing thread properties}

Two commercial sewing threads, commonly used for sewing denim fabrics, were chosen. The linear density of sewing thread was chosen according to NFG 07-117 [18]. Note that two types of "Royal" sewing threads were used. They were twotwisted and three-twisted polyester spun threads. The actual counts of the threads were 63.5 tex and 95 tex.

The seam threads' properties are shown in Table 2.

To ensuring the same conditions for all tests, cover stitch machines type MO-3316 parameters were not only adjusted to the manufacturer's recommended standard settings but also were kept constant during experimentations and the same human skills and process, etc. are used. Thus, to measure the sewing thread consumption of all denim samples, our study starts with developing all relationships using studied stitch types. Based on the earlier works [1, 15-17, 19-21], the chosen seam length per test is $100 \mathrm{~mm}$.

Table 2. Sewing threads properties

\begin{tabular}{|c|c|c|}
\hline Properties & $\begin{array}{c}\text { Thread } \\
\text { No 1 }\end{array}$ & $\begin{array}{c}\text { Thread } \\
\text { No 2 }\end{array}$ \\
\hline Linear density (tex) & 63.50 & 95.00 \\
\hline Number of strands & 3 & 3 \\
\hline Composition & $100 \%$ PES & $100 \%$ PES \\
\hline Twist direction & S & S \\
\hline Breaking force (N) & 24.23 & 26.43 \\
\hline Breaking elongation (\%) & 21.12 & 16.90 \\
\hline Rigidity (N/m) & 389.12 & 536.59 \\
\hline Twist/m & 353.00 & 294.00 \\
\hline Tenacity (cN/tex) & 38.16 & 27.82 \\
\hline
\end{tabular}

With PES: Means polyester material.

\subsection{Methods used for thread consumption prediction}

A geometrical method and a multi-linear regression method are considered in this work. We tested the mostly used cover stitch class 600 in sewn product industry. In fact, stitch types are classified into six classes ranging from class 100 to class 600 according to the ASTM standard. These classes are classified as follows; single thread chain stitch (class 100), hand stitch (class 200), lockstitch (class 300), multiple thread chain stitch (class 400), overlock stitch (class 500), and stitch cover (class 600). The seam class determination depends on the configuration and the mechanism of entanglement and interlacement of threads.

Cover stitch class 600 is the class commonly used in most types of sewn products. Its thread consumption is as high as class 400 . The types of stitches 600 are simple recovering stitches to which an upper cover thread is added using an upper covering device or a looper. This upper cover is used for aesthetic purposes and to protect the upper surface of the seam, if necessary. As this is flat and comfortable to use, this is often used for stretch clothing. It is also used for decorative stitching because of its superior cover wire. Over the years, the over stitch 606 has been largely replaced by the over stitch 607 . This stitch type is formed with four needle threads, a looper thread, and an overlay thread. Cover stitch type 607 requires a large amount of thread but it has the advantage of creating flat, comfortable, and highly extensible seams. Further, three types of stitch generally used are chosen. In our work, we try to establish developed models to determine consumed thread based on cover stitch class 600 , thus, making our present study a true and original one, because until now there is no work has dealt with overall types of stitch in the same class.

Note that three types of stitch for the class 600 were considered. These stitches have double recovery (lower and upper):

Cover stitch 602: Two needles, four threads;

\section{Chain stitch 605: Three needles, five threads; and}

Chain stitch 607: four needles, six threads.

Nevertheless, for the multi-linear regression, a statistical method based on experimental design was applied to conduct experiments on this modeling technique using MINITAB-17 software. Moreover, based on these multi-linear regression models, thanks to MINITAB-17 software, individual effects of input parameters were determined, analyzed, and classified. Five input parameters with their levels were taken into account (material thickness (6 levels), stitch density [3 levels], yarn count [2 levels], seam width [2 levels], and gap between two needles [2 levels]) for both geometrical and statistical methods. It is highly complicated to optimize all effective parameters during the sewing process. The predicted thread consumption is still related to the stitched fabric plies or layers. Note that all samples are sewn using two fabric plies in this work. 


\section{Results and discussion}

\subsection{Geometrical model}

Mathematical calculations based on geometrical shapes of cover stitch geometries were used to realize the stitch modeling. Some assumptions were considered to facilitate the calculation of the consumed thread. To represent overall shape geometries of cover stitch class 600 , we used two software namely 3ds Max 2014 and keyshot 5 . The cross -section of threads is circular and incompressible in nature in our case. In addition, the same thread was used in all cases (needle, upper, and lower loopers). Also, the diameters of needle and looper(s) thread are equal; these are also equal to yarn present in a given fabric. The fabric is also incompressible in nature. The model is three dimensional in nature.

using the geometrical representation of each studied chain stitch, the length of the sewing thread (from needle threads [n1 and n2 having red color]) and looper threads (blue and yellow threads) were calculated for each stitch based on simple mathematical principles (sum, shape calculation using basic mathematical relationships, circular section of thread, incompressible threads and incompressible fabrics, etc.) In addition, a unit cell of a seam is of rectangular shape having width equal to $\frac{1}{-}$. The thread length of a unit cell is assumed to be the same throughout the seam length for overall repeated unit.

So, single thread consumption is calculated separately for each unit cell. In fact, simply we follow the thread trajectory and used values and parameters are as follows;

- $\quad S L$ distance is considered in lengthways

- MT distance is considered in height way

- $\quad B_{1}, B_{2}$ and $B_{3}$ are considered distances in width way

- In the other cases, mathematical equations are considered. For example to determine the $c$ distance value (Figure 1) the following equation is considered: $a^{2}+b^{2}=c^{2}$

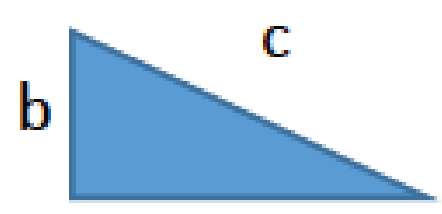

a

Figure 1. Example of determining $\mathrm{c}$ distance value.

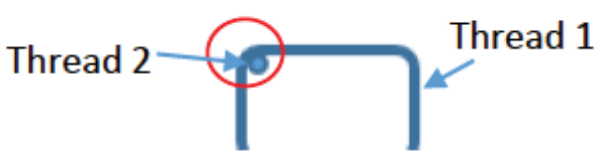

Figure 2. Example 1 of curvature.

\section{Thread 2 Thread 1}

Figure 3. Example 2 of curvature.

- Thread diameter value $d$ is added with any value if it has impact in the trajectory

- The curvatures; we have different ways to determinate the curvature value, for example for thread 1 consumption in curvature:

According to Figure 2, thread consumption is equal to $\frac{1}{4}\left(\pi \times\left(d+\frac{d}{2}\right)\right)$.

When referring to Figure 3 , thread consumption in this case is equal to $d$.

\subsubsection{Case of cover stitch type 602}

A double stitch consists of complex interlacing of looper and needle threads. By investigating the shape of 602 cover stitch, it was found that different parts could be separately investigated successfully to calculate the total amount of the stitch during seaming $\left(C_{n 1602}, C_{n 2602}, C_{u l 602}\right.$, and $\left.C_{\| l 602}\right)$. Figure 4 indicates the actual seam configuration and the positions of interlacing points formed by needles and loopers threads.

So, sewing thread consumption to make a single stitch can be calculated by using Eq. (1).

The amount of sewing thread $C_{602}$ needed for the 602 cover stitch, whose geometry is represented previously in Figure 4, is estimated by the following formula 1 .

$C_{602}=\left(C_{n 1602}+C_{n 2602}+C_{u 1602}+C_{11602}\right) \times n \times L$

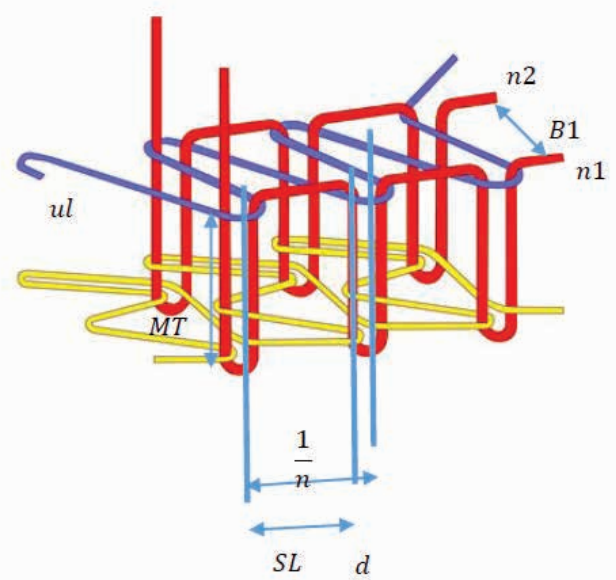

Figure 4. Geometrical model of cover stitch type 602 presented in 3 Dimensions 
Where: $C_{n 1602}$ is the consumption of needle 1 thread, $C_{n 2602}$ is the consumption of needle 2 thread, $C_{u l 602}$ is the consumption of upper looper thread, and $C_{\| / 602}$ is the consumption of upper looper thread.

Where:

$$
\begin{aligned}
& C_{n 1602}=S L+2 M T+\frac{1}{2}\left(\pi \times\left(d+\frac{d}{2}\right)\right) 2 \times \frac{d}{2} \\
& S L+2 M T+d\left(\frac{3}{2} \pi+1\right)
\end{aligned}
$$

Where $C_{n 1602}=C_{n 2602}$

$$
\begin{aligned}
& C_{u l ~ 602}=B_{1}+\sqrt{B_{1}^{2}+S L^{2}}+2 \times\left(\frac{1}{2}\left(\pi \times\left(d+\frac{d}{2}\right)\right)\right) \\
& C_{l l 602}=3 \times \frac{1}{n}+S L+\left(\left(\frac{1}{n}\right)^{2}+B_{1}^{2}\right)^{1 / 2}+B_{1}+4 \times \frac{1}{2} \times\left(\pi \times\left(d+\frac{d}{2}\right)\right)+2 \times \frac{d}{2}
\end{aligned}
$$

We used the general equation supposed by Sohanur [22] as a function of the thread linear density Tex to calculate a sewing thread diameter supposed as circular (d) (Eq. 4).

$d(\mathrm{~cm}) \frac{0.375}{100} \sqrt{\mathrm{Tex}}$

\subsubsection{Case of cover stitch type 605}

When investigating the shape of 605 cover stitch, it was found that different parts can be separately investigated successfully to calculate the total amount of the stitch during seaming $\left(C_{n 1}\right.$ ${ }_{605}, C_{n 2605}, C_{n 3605}, C_{u 1605}$, and $\left.C_{u 1605}\right)$. This type of stitch is formed with five threads: three needle threads, a looper thread, and an upper thread. 605-stitch type produces very elastic seams, and requires a larger average amount of yarn.

The amount of sewing thread $C_{605}$ needed for the 605 chain stitch, whose geometry is represented previously in Figure 5, is estimated by the following Equation. (7).

$C_{665}=\left(C_{n 1605}+C_{n 2605}+C_{n 3605}+C_{w 605}+C_{n 16605}\right) \times n \times L$

where: $C_{n 1605}, C_{n 2605}$ and $C_{n 3605}$ are the consumption thread of needle 1,2 , and 3 , respectively. Moreover, $C_{u l 605}$ and $C_{u l}$ ${ }_{605}$ are the consumptions threads of upper and looper threads, respectively.

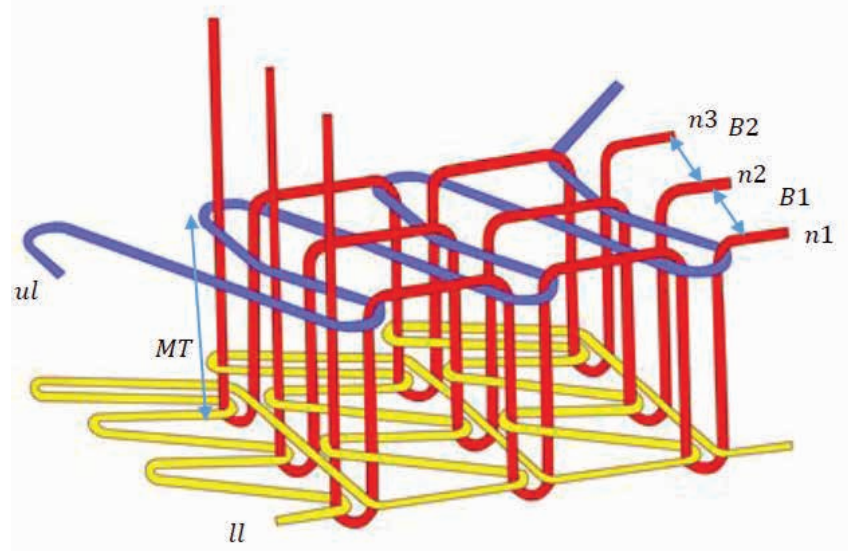

Figure 5. Geometrical model of cover stitch type 605 presented in 3 Dimensions
Table 3. Different factor for each type of Chain stitch

\begin{tabular}{|c|c|c|c|c|}
\hline Chain stitches & $\boldsymbol{n}$ & $\boldsymbol{d}$ & $\mathbf{M T}$ & $\boldsymbol{B}_{\mathbf{1}}$ \\
\hline 602 & $*$ & $*$ & $*$ & $*$ \\
\hline 605 & $*$ & $*$ & $*$ & \\
\hline
\end{tabular}

${ }^{*}$ Represents the existent parameter for each studied chain stitch type. where $B_{2}=0.3 \mathrm{~cm}$

Table 4. Levels of each factor

\begin{tabular}{|c|c|c|c|c|}
\hline $\begin{array}{c}\text { Input } \\
\text { parameter }\end{array}$ & $\begin{array}{c}\boldsymbol{n} \\
\mathbf{( s t i t c h e s /} \\
\mathbf{c m})\end{array}$ & $\boldsymbol{d} \mathbf{( \mathbf { c m } )}$ & $\begin{array}{c}\mathbf{M T} \\
\mathbf{( c m )}\end{array}$ & $\mathbf{B}_{1} \mathbf{( c m )}$ \\
\hline Level 1 & 3 & 0.024 & 0.240 & 0.3 \\
\hline Level 2 & 4 & 0.036 & 0.208 & 0.6 \\
\hline Level 3 & 5 & $*-$ & 0.192 & - \\
\hline Level 4 & - & - & 0.180 & - \\
\hline Level 5 & - & - & 0.170 & - \\
\hline Level 6 & - & - & 0.150 & - \\
\hline
\end{tabular}

*No value of parameter, which should be mentioned.

$$
\begin{aligned}
& C_{605}=S L+2 M T+\frac{1}{2}\left(\pi \times\left(d+\frac{d}{2}\right)\right)+2 \times \frac{d}{2} \\
& =S L+2 M T+d\left(\frac{3}{2} \pi+1\right)
\end{aligned}
$$

where $C_{n 1605}=C_{n 2605}=C_{n 2605}$

$$
\begin{aligned}
& C_{u l ~ 605}=B_{2}+\sqrt{\left(B_{1}+B_{2}\right)^{2}+S L^{2}}+\sqrt{B_{1}^{2}+(3 d)^{2}}+2 \times\left(\pi \times\left(d+\frac{d}{2}\right)\right) \\
& 4 \times \frac{1}{n}+S L+\left(\left(\frac{1}{n}\right)^{2}+B_{1}^{2}\right)^{1 / 2}+\left(\left(\frac{1}{n}\right)^{2}+B_{2}^{2}\right)^{1 / 2}+B_{1}+B_{2}+6 \times \frac{1}{n} \times\left(\pi \times\left(d+\frac{d}{2}\right)\right)+2 \times \frac{d}{2}
\end{aligned}
$$

\subsubsection{Case of cover stitch type 607}

Based upon the geometrical model of cover stitch type 607, the different parts that are investigated to calculate the total amount of the stitch are $C_{n 1607}, C_{n 2607}, C_{n 3607}, C_{n 4607}, C_{u 1607}$, and $C_{u l 607}$. The amount of sewing thread $C_{607}$ needed for the 607 chain stitch, whose geometry is represented previously in Figure 6, is estimated by the following formula 12 .

$C_{607}=\left(C_{n 1607}+C_{n 2607}+C_{n 3607}+C_{n 4607}+C_{u l 607}+C_{u l 607}\right) \times n \times L$

where: $C_{n 1607}, C_{n 2607}, C_{n 3607}$, and $C_{n 4607}$ are the consumption thread of needle 1,2 , and 3 and 4 , respectively. Moreover, $C_{u l}$ ${ }_{607}$ and $C_{u l 605}$ are the consumptions thread of upper and looper threads, respectively.

$$
\begin{aligned}
& C_{n 1607}=S L+2 M T+\frac{1}{2}\left(\pi \times\left(d+\frac{d}{2}\right)\right)+2 \times \frac{d}{2} \\
& =S L+2 M T+d\left(\frac{3}{2} \pi+1\right)
\end{aligned}
$$

where $C_{n 1607}=C_{n 2607}=C_{n 3607}=C_{n 4607}$ 
Table 5. Compared experimental and theoretical results for 602, 605, and 607

\begin{tabular}{|c|c|c|c|c|c|c|c|c|c|}
\hline \multicolumn{3}{|c|}{ Input parameters } & \multirow[b]{2}{*}{$B_{1}$} & \multicolumn{3}{|c|}{602} & \multicolumn{3}{|c|}{605} \\
\hline $\begin{array}{c}n \\
\text { (stitches/cm) }\end{array}$ & $d(\mathrm{~cm})$ & MT & & $C_{\mathrm{Th}}(\mathrm{cm})$ & $C_{\operatorname{Exp}}(\mathrm{cm})$ & Error & $C_{\mathrm{Th}}(\mathrm{cm})$ & $C_{\operatorname{Exp}}(\mathrm{cm})$ & Error \\
\hline 3 & 0.024 & 0.240 & 0.3 & 12.23 & 11.76 & $4.00 \%$ & 23.35 & 21.82 & $7.01 \%$ \\
\hline 3 & 0.024 & 0.208 & 0.3 & 12.04 & 11.51 & $4.60 \%$ & 22.78 & 21.67 & $5.12 \%$ \\
\hline 3 & 0.024 & 0.192 & 0.3 & 11.94 & 11.72 & $1.88 \%$ & 22.49 & 21.61 & $4.07 \%$ \\
\hline 3 & 0.024 & 0.180 & 0.3 & 11.87 & 11.62 & $2.15 \%$ & 22.27 & 21.64 & $2.91 \%$ \\
\hline 3 & 0.024 & 0.170 & 0.3 & 11.81 & 11.71 & $0.85 \%$ & 22.09 & 21.73 & $1.66 \%$ \\
\hline 3 & 0.024 & 0.150 & 0.3 & 11.69 & 11.26 & $3.82 \%$ & 21.73 & 21.26 & $2.21 \%$ \\
\hline 4 & 0.024 & 0.240 & 0.3 & 14.23 & 13.45 & $5.80 \%$ & 27.87 & 26.21 & $6.33 \%$ \\
\hline 4 & 0.024 & 0.208 & 0.3 & 13.98 & 13.41 & $4.25 \%$ & 27.10 & 25.81 & $5.00 \%$ \\
\hline 4 & 0.024 & 0.192 & 0.3 & 13.85 & 13.25 & $4.53 \%$ & 26.72 & 25.61 & $4.33 \%$ \\
\hline 4 & 0.024 & 0.180 & 0.3 & 13.75 & 13.75 & $0.00 \%$ & 26.43 & 25.53 & $3.53 \%$ \\
\hline 4 & 0.024 & 0.170 & 0.3 & 13.67 & 13.36 & $2.32 \%$ & 26.19 & 25.22 & $3.85 \%$ \\
\hline 4 & 0.024 & 0.150 & 0.3 & 13.51 & 12.91 & $4.65 \%$ & 25.71 & 25.19 & $2.06 \%$ \\
\hline 5 & 0.024 & 0.240 & 0.3 & 16.29 & 15.49 & $5.16 \%$ & 32.46 & 30.93 & $4.95 \%$ \\
\hline 5 & 0.024 & 0.208 & 0.3 & 15.97 & 15.65 & $2.04 \%$ & 31.50 & 30.01 & $4.97 \%$ \\
\hline 5 & 0.024 & 0.192 & 0.3 & 15.81 & 15.45 & $2.33 \%$ & 31.02 & 29.95 & $3.57 \%$ \\
\hline 5 & 0.024 & 0.180 & 0.3 & 15.69 & 15.60 & $0.58 \%$ & 30.66 & 29.50 & $3.93 \%$ \\
\hline 5 & 0.024 & 0.170 & 0.3 & 15.59 & 15.47 & $0.78 \%$ & 30.36 & 29.68 & $2.29 \%$ \\
\hline 5 & 0.024 & 0.150 & 0.3 & 15.39 & 15.26 & $0.85 \%$ & 29.76 & 29.20 & $1.92 \%$ \\
\hline 3 & 0.036 & 0.240 & 0.3 & 12.85 & 12.21 & $5.24 \%$ & 24.70 & 23.51 & $5.06 \%$ \\
\hline 3 & 0.036 & 0.208 & 0.3 & 12.66 & 12.30 & $2.93 \%$ & 24.12 & 22.78 & $5.88 \%$ \\
\hline 3 & 0.036 & 0.192 & 0.3 & 12.57 & 12.54 & $0.24 \%$ & 23.84 & 22.71 & $4.98 \%$ \\
\hline 3 & 0.036 & 0.180 & 0.3 & 12.49 & 12.16 & $2.71 \%$ & 23.62 & 22.75 & $3.82 \%$ \\
\hline 3 & 0.036 & 0.170 & 0.3 & 12.43 & 12.12 & $2.56 \%$ & 23.44 & 22.84 & $2.63 \%$ \\
\hline 3 & 0.036 & 0.150 & 0.3 & 12.31 & 11.66 & $5.57 \%$ & 23.08 & 22.70 & $1.67 \%$ \\
\hline 4 & 0.036 & 0.240 & 0.3 & 15.09 & 14.19 & $6.34 \%$ & 29.67 & 28.16 & $5.36 \%$ \\
\hline 4 & 0.036 & 0.208 & 0.3 & 14.83 & 14.47 & $2.49 \%$ & 28.90 & 27.78 & $4.03 \%$ \\
\hline 4 & 0.036 & 0.192 & 0.3 & 14.71 & 14.30 & $2.87 \%$ & 28.52 & 27.59 & $3.37 \%$ \\
\hline 4 & 0.036 & 0.180 & 0.3 & 14.61 & 14.51 & $0.69 \%$ & 28.23 & 27.52 & $2.58 \%$ \\
\hline 4 & 0.036 & 0.170 & 0.3 & 14.53 & 14.41 & $0.83 \%$ & 27.99 & 27.22 & $2.83 \%$ \\
\hline 4 & 0.036 & 0.150 & 0.3 & 14.37 & 14.20 & $1.20 \%$ & 27.51 & 26.90 & $2.27 \%$ \\
\hline 5 & 0.036 & 0.240 & 0.3 & 17.38 & 16.50 & $5.33 \%$ & 34.72 & 32.37 & $7.26 \%$ \\
\hline 5 & 0.036 & 0.208 & 0.3 & 17.06 & 16.39 & $4.09 \%$ & 33.76 & 31.72 & $6.43 \%$ \\
\hline 5 & 0.036 & 0.192 & 0.3 & 16.90 & 16.48 & $2.55 \%$ & 33.28 & 31.40 & $5.99 \%$ \\
\hline 5 & 0.036 & 0.180 & 0.3 & 16.78 & 16.64 & $0.84 \%$ & 32.92 & 31.22 & $5.45 \%$ \\
\hline 5 & 0.036 & 0.170 & 0.3 & 16.68 & 16.51 & $1.03 \%$ & 32.62 & 30.85 & $5.74 \%$ \\
\hline 5 & 0.036 & 0.150 & 0.3 & 16.48 & 16.37 & $0.67 \%$ & 32.02 & 31.50 & $1.65 \%$ \\
\hline 3 & 0.024 & 0.240 & 0.6 & 15.37 & 15.06 & $2.06 \%$ & ${ }^{*}-$ & - & - \\
\hline
\end{tabular}


Table 5 continued. Compared experimental and theoretical results for 602,605 , and 607

\begin{tabular}{|c|c|c|c|c|c|c|c|c|c|}
\hline \multicolumn{3}{|c|}{ Input parameters } & \multirow[b]{2}{*}{$\mathrm{B}_{1}$} & \multicolumn{3}{|c|}{602} & \multicolumn{3}{|c|}{605} \\
\hline $\begin{array}{c}n \\
\text { (stitches/cm) }\end{array}$ & $d(\mathrm{~cm})$ & $M T$ & & $C_{\mathrm{Th}}(\mathrm{cm})$ & $C_{\text {Exp }}(\mathrm{cm})$ & Error & $C_{\mathrm{Th}}(\mathrm{cm})$ & $C_{\text {Exp }}(\mathrm{cm})$ & Error \\
\hline 3 & 0.024 & 0.208 & 0.6 & 15.17 & 14.87 & $2.02 \%$ & - & - & - \\
\hline 3 & 0.024 & 0.192 & 0.6 & 15.08 & 14.73 & $2.38 \%$ & - & - & - \\
\hline 3 & 0.024 & 0.180 & 0.6 & 15.01 & 14.92 & $0.60 \%$ & - & - & - \\
\hline 3 & 0.024 & 0.170 & 0.6 & 14.95 & 14.83 & $0.81 \%$ & - & - & - \\
\hline 3 & 0.024 & 0.150 & 0.6 & 14.83 & 14.70 & $0.88 \%$ & - & - & - \\
\hline 4 & 0.024 & 0.240 & 0.6 & 18.55 & 17.63 & $5.22 \%$ & - & - & - \\
\hline 4 & 0.024 & 0.208 & 0.6 & 18.30 & 17.77 & $2.98 \%$ & - & - & - \\
\hline 4 & 0.024 & 0.192 & 0.6 & 18.17 & 18.13 & $0.22 \%$ & - & - & - \\
\hline 4 & 0.024 & 0.180 & 0.6 & 18.07 & 17.59 & $2.73 \%$ & - & - & - \\
\hline 4 & 0.024 & 0.170 & 0.6 & 17.99 & 17.50 & $2.80 \%$ & - & - & - \\
\hline 4 & 0.024 & 0.150 & 0.6 & 17.83 & 17.71 & $0.68 \%$ & - & - & - \\
\hline 5 & 0.024 & 0.240 & 0.6 & 21.79 & 21.60 & $0.88 \%$ & - & - & - \\
\hline 5 & 0.024 & 0.208 & 0.6 & 21.47 & 21.21 & $1.23 \%$ & - & - & - \\
\hline 5 & 0.024 & 0.192 & 0.6 & 21.31 & 20.24 & $5.29 \%$ & - & - & - \\
\hline 5 & 0.024 & 0.180 & 0.6 & 21.19 & 20.36 & $4.08 \%$ & - & - & - \\
\hline 5 & 0.024 & 0.170 & 0.6 & 21.09 & 20.67 & $2.03 \%$ & - & - & - \\
\hline 5 & 0.024 & 0.150 & 0.6 & 20.89 & 20.28 & $3.01 \%$ & - & - & - \\
\hline 3 & 0.036 & 0.240 & 0.6 & 15.96 & 15.65 & $1.98 \%$ & - & - & - \\
\hline 3 & 0.036 & 0.208 & 0.6 & 15.77 & 15.16 & $4.02 \%$ & - & - & - \\
\hline 3 & 0.036 & 0.192 & 0.6 & 15.67 & 15.29 & $2.49 \%$ & - & - & - \\
\hline 3 & 0.036 & 0.180 & 0.6 & 15.60 & 15.57 & $0.19 \%$ & - & - & - \\
\hline 3 & 0.036 & 0.170 & 0.6 & 15.54 & 15.09 & $2.98 \%$ & - & - & - \\
\hline 3 & 0.036 & 0.150 & 0.6 & 15.42 & 14.83 & $3.98 \%$ & - & - & - \\
\hline 4 & 0.036 & 0.240 & 0.6 & 19.36 & 18.96 & $2.11 \%$ & - & - & - \\
\hline 4 & 0.036 & 0.208 & 0.6 & 19.10 & 18.67 & $2.30 \%$ & - & - & - \\
\hline 4 & 0.036 & 0.192 & 0.6 & 18.97 & 18.24 & $4.00 \%$ & - & - & - \\
\hline 4 & 0.036 & 0.180 & 0.6 & 18.88 & 18.33 & $3.00 \%$ & - & - & - \\
\hline 4 & 0.036 & 0.170 & 0.6 & 18.80 & 18.34 & $2.51 \%$ & - & - & - \\
\hline 4 & 0.036 & 0.150 & 0.6 & 18.64 & 18.09 & $3.04 \%$ & - & - & - \\
\hline 5 & 0.036 & 0.240 & 0.6 & 22.79 & 22.13 & $2.98 \%$ & - & - & - \\
\hline 5 & 0.036 & 0.208 & 0.6 & 22.47 & 21.86 & $2.79 \%$ & - & - & - \\
\hline 5 & 0.036 & 0.192 & 0.6 & 22.31 & 21.75 & $2.57 \%$ & - & - & - \\
\hline 5 & 0.036 & 0.180 & 0.6 & 22.19 & 21.30 & $4.18 \%$ & - & - & - \\
\hline 5 & 0.036 & 0.170 & 0.6 & 22.09 & 21.37 & $3.37 \%$ & - & - & - \\
\hline 5 & 0.036 & 0.150 & 0.6 & 21.89 & 21.61 & $1.30 \%$ & - & - & - \\
\hline
\end{tabular}

"No value saved. 


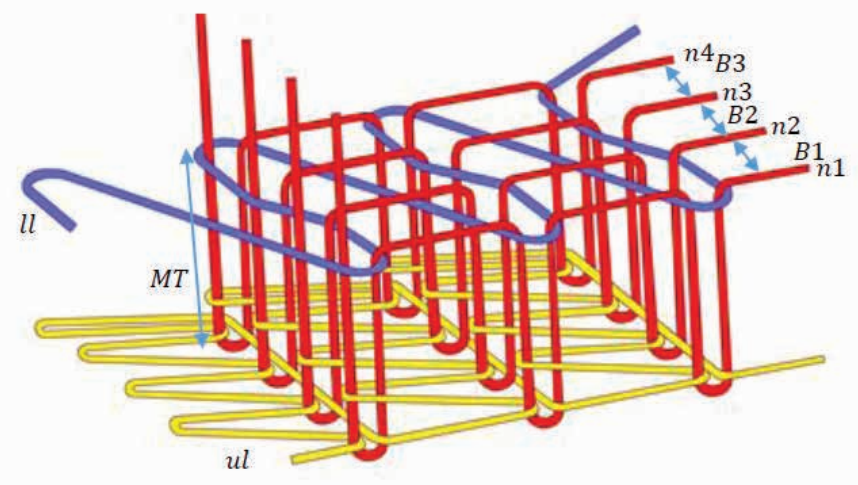

Figure 6. Geometrical model of cover stitch type 607 presented in 3 Dimensions

$$
\begin{aligned}
& C_{\text {wl } 514}=B_{1}+B_{3}+\sqrt{\left(B_{1}+B_{2}+B_{3}\right)^{2}+S L^{2}}+\sqrt{\left(B_{2}\right)^{2}+(3 d)^{2}}+2 \times\left(\pi \times\left(d+\frac{d}{x}\right)\right)
\end{aligned}
$$

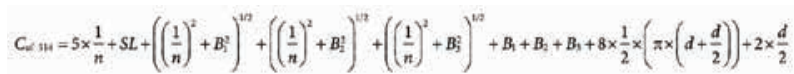

Please note that 72 samples were prepared by varying the different factors for cover stitch 602 and 36 samples were prepared for cover stitch 605 for verification of these models.

The industrial sewing machine type SIRUBA F007 (WL22-356) (Speed: $2000 \mathrm{rpm} / \mathrm{min}, 3$ needles) was used for both cover stitch types 602 and 605.

Table 3 presents the different factors and levels for each type of cover stitch point.

Table 4 presents the levels of each factor for preparing the samples of each type of cover stitch class 600 .

Each sample with $10 \mathrm{~cm}$ seam length was investigated. Then, the seam was unstitched to get the needle; upper looper and lower looper thread consumed in $5 \mathrm{~cm}$ length. After unstitching, the sewed thread length was determined to measure the value of the consumed thread per $\mathrm{cm}$.

Table 5 presents a comparison between the experimental $\left(C_{\text {Exp }}\right)$ and the theoretical $\left(C_{\mathrm{Th}}\right)$ thread consumptions within their absolute errors based on the cover stitch type 602 and 605.

The average absolute relative error, $\overline{\text { Error }}$ (Eq. 23) between the theoretical $\left(C_{\mathrm{Th}}\right)$ and experimental values $\left(C_{\mathrm{Exp}}\right)$ was determined to improve the obtained results. The limit of the mean absolute error values used in the modeling is estimated to be $6 \%$ [23].

Table 7. Multi-linear regression models for different cover stitch class 600

\begin{tabular}{|c|c|}
\hline Equation & $\boldsymbol{R}^{\mathbf{2}} \mathbf{( \% )}$ \\
\hline$C_{602}=-3.414+2.5611 n+65.38 d+4.88 \mathrm{MT}+14.020 \mathrm{~B}_{1}$ & 97.05 \\
\hline$C_{605}=3.376+4.2217 n+136.28 d+11.54 \mathrm{MT}$ & 99.63 \\
\hline
\end{tabular}

Table 6. Average error

\begin{tabular}{|l|l|l|}
\hline Stitch & $\mathbf{4 0 1}$ & $\mathbf{4 0 2}$ \\
\hline$\overline{\text { Error }(\%)}$ & 2.60 & 4.07 \\
\hline
\end{tabular}

$\overline{\text { Error }}=\frac{\left|C_{\mathrm{Th}}-C_{\mathrm{Exp}}\right|}{C_{\mathrm{Exp}}} \times 100$

Table 6 presents the average error for each type of cover stitch class 600 .

The average absolute error does not exceed $4.07 \%$, which means the result of testing thread consumption is widely verified. Regarding the findings, the error values ( $\overline{\text { Error }}(\%)$ ) are much lower than $6 \%$. Thus, the studied models are well justified and the findings are highly significant.

Comparing the experimental and theoretical values showed a good agreement between geometrical and experimental consumption values. Moreover, the coefficient of regression ranged from $98.78 \%$ to $99.38 \%$ (Figure 4), which reflects significant and efficient relationships between experimental and theoretical findings.

Comparisons between the theoretical and experimental consumption values for cover stitch type 602 and 605 are shown in Figures $4 \mathrm{a}$ and $4 \mathrm{~b}$. Experimental thread consumption ( $x$-axis) is plotted against theoretical thread consumption ( $y$-axis). Linear regression is applied and a line is fitted to the data. The $R^{2}$ value of the fitted line is ranging from $98.78 \%$ to $99.38 \%$, which means that the difference between the two data sets is insignificant. The high value of $R^{2}$ verifies the model accuracy and depicts that the model has taken into account all the variables on which the thread consumption depends. This result is similar to Abher who used geometrical models and concluded that the difference between the actual and predicted thread consumption, for 504 over edge stich, sets is insignificant ( $R^{2}$ value of the fitted line is 0.992$)$ [11].

As the error rate is very low and the regression coefficients values are very high $(98.78 \%-99.38 \%)$, it was concluded that the obtained geometrical models are worthwhile and effective, and could be recommended for the prediction of thread consumption in the experimental design.

The results obtained for cover stitch types 602 and 605, respectively, are effectively proved and sufficiently by applying geometrical technique. Based on these findings, it was concluded that the proposed models of cover stitch type 607 is also successfully proved.

Table 8. $p$-value for each studied model

\begin{tabular}{|c|c|c|c|c|}
\hline Parameter & $\boldsymbol{n}$ & $\boldsymbol{d}$ & MT & $\boldsymbol{B}_{1}$ \\
\hline 602 model & 0.000 & 0.000 & 0.020 & 0.000 \\
\hline 605 model & 0.000 & 0.000 & 0.000 & ${ }^{*}$ \\
\hline
\end{tabular}

*No value of parameter which should be mentioned. 


\subsection{Multi-linear regression models}

The multi-linear regression method was performed and the results are compared with the results obtained using the geometrical method to help industrialists to select and use the best method to predict the consumption of thread to sew denim garments,

Based on experimental design, a statistical method was applied to study the effect of each investigated input parameter and accurately estimate the suitable amount of thread based on different cover stitch types. Using the MINITAB-17 software, the multi-linear regression models were determined. Each input parameter effects of are analyzed. The studied input parameters as well as their levels are presented in Tables 3 and 4 , respectively. The different models for cover stitch types 602 and 605 are considered as shown in Table 7.

The value of regression coefficient $R^{2}$ reflects the significance and relevance of the developed models. Indeed, this coefficient of regression (Eq. 24) ranged from $0 \%$ to $100 \%$. The closer it is to $100 \%$, the more significant the model is and vice versa [1].

$R^{2}=1-\frac{\mathrm{SS}_{\text {error }}}{\mathrm{SST}}=1-\frac{\mathrm{SS}_{\mathrm{reg}}}{\mathrm{SST}}$

where:

SS $_{\text {error }}$ : The portion of the variation, which not explained by the model and is attributed to error. It is calculated according to Eq. (25):

$\mathrm{SS}_{\text {error }}=\sum\left(v_{i}-\hat{v}\right)^{2}$

SS $_{\text {error }}$ The portion explained by the multi-linear regression model. We used Eq (26) to calculate the value:

$\mathrm{SS}_{\text {reg }}=\sum\left(\hat{v}_{i}-\bar{v}\right)^{2}$

SST: The sum of squared distances represents the total variation in the experimental data according to these relationships (Eq. 27):

$\mathrm{SST}=\mathrm{SS}_{\text {reg }}+\mathrm{SS}_{\text {error }}$

However, it is important to analyze the statistical analysis variance. Based on the $p$-value statistical coefficient, it is possible to evaluate the importance of different parameters. Indeed, three conditions are presented:

- $\quad p$-value is null: parameter very significant.

- $\quad p$-value is ranged from 0 to 0.05 : significant parameter.

- $\quad p$-value is higher than 0.05: negligible parameter.

Table 8 presents the $p$-values for each multi-linear regression model.

Each parameter with a $p$-value higher than 0.05 is neglected. Therefore, referring to Table 8, not all parameters are neglected and the multi-linear regression models presented in Table 7 are considered.

Considering the range of $R^{2}$ values obtained (from $97.05 \%$ to $99.63 \%$ ), it is considered as reliable and significant. Therefore, this range of $R^{2}$ shows the effectiveness of the statistical method to determine the suitable consumption value. Moreover, to predict consumed thread using multi-linear regression models, industrialists can use statistical results for the estimation of the consumed amount. Nevertheless, compared to geometrical modeling technique, statistical one remained less accurate, powerful, and predictable because of the range of $R^{2}$ (from $98.78 \%$ to $99.38 \%$ and from $97.05 \%$ to $99.63 \%$, for geometrical and statistical models, respectively). In fact, the result obtained by using geometrical presentation is precise because this method based on a pure mathematical calculation. However, statistical method is based on practical tests where the uncertainties of measurements (environment, equipment, etc.) are probable.

However, the geometrical and statistical results are significant and can predict the seam thread consumption. When compared with regressive and geometrical methods, the geometrical method is found to be more effective than the regressive method. However, the difference is not very important. So we can consider that the two models can widely predict seam thread consumption value. The developed geometrical and statistical models allow a successful prediction of the consumed thread amount for the experimental design of interest. These findings seem to be in good agreement with those in the literature survey $[6,11]$.

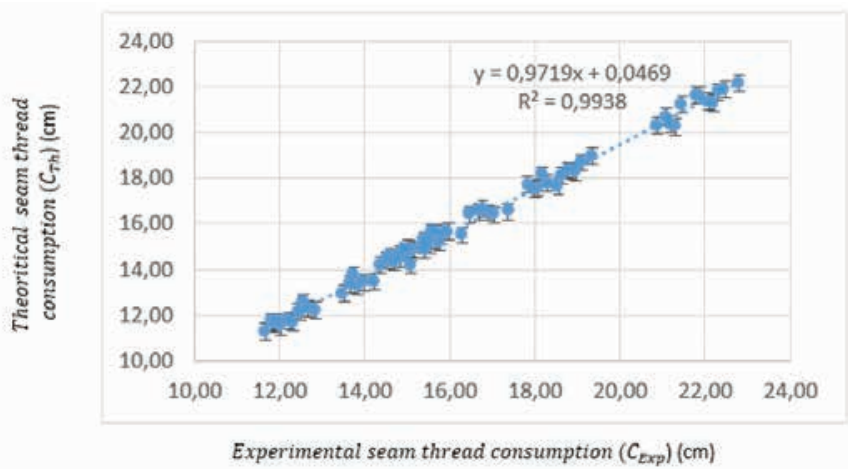

(a)

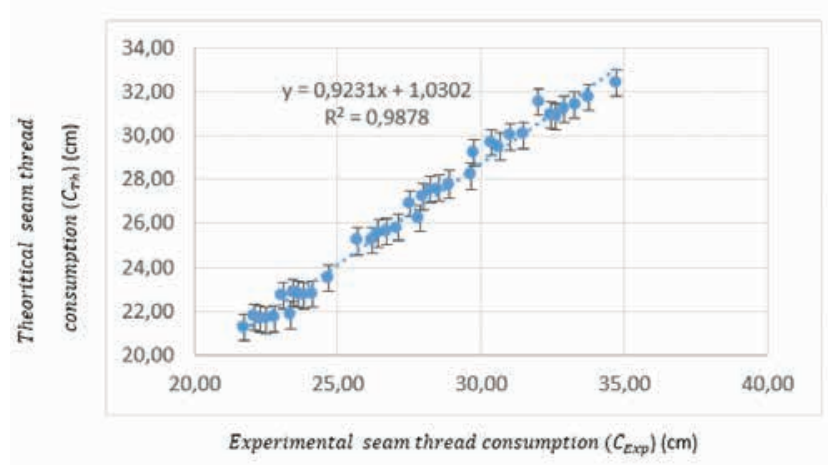

(b)

Figure 7. Theoretical consumption evaluation as a function of experimental results for cover stitch type 602 (a) and 605 (b) 


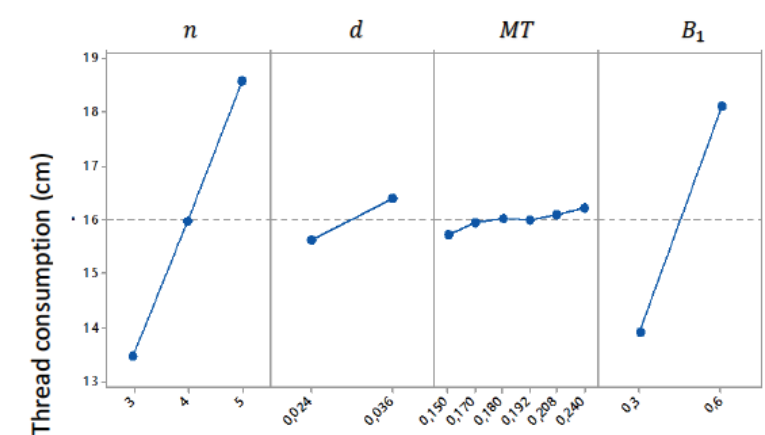

(a)

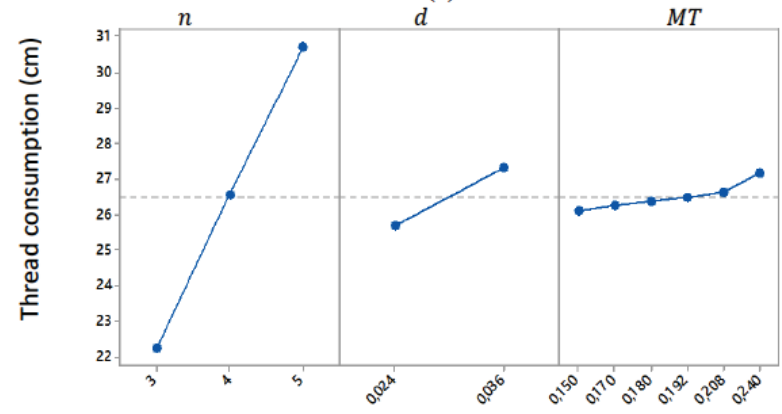

(b)

Figure 8. Effects of input parameters for cover stitch type 602 (a) and 605 (b)

\subsubsection{Effects of input parameters}

After determining the regression models, the effect of each input parameter is determined. Figure 8 presents the influence of material thickness, stitch density, yarn linear density, and the seam width for the different stitches (602 and 605).

\subsubsection{Stitch density effect}

Considering the Figures $8 \mathrm{a}$ and $8 \mathrm{~b}$, stitch density is found to be the most important parameter. In fact, the increase of stitch density from 3 to 5 stiches/cm increases the thread consumption percentage by $38.01 \%$ and $37.93 \%$ for 602 and 605 cover stitches, respectively. Thus, the increase of stitch density increases thread consumption. Besides, the increase of the consumption amount of sewing thread results from the increase in interlacing zone number inside assembled fabric layers. This result is in accordance with the findings of Jaouachi, Kennon, Hayes, and Abher [6, 24-26] and confirms our finding, i.e., the quantity of thread required when the fabric feed in motion to form the upper and lower lengths during stitch length modification. Moreover, this result is in good agreement with the findings of Lauriol's study [4] which proves that if the sewing length decreases from $2.5 \mathrm{~mm}$ to $2 \mathrm{~mm}$ (from 4 stitches $/ \mathrm{cm}$ to 5 stitches $/ \mathrm{cm}$ ), thread consumption increases, approximately, by $10 \%$. According to Sharma study, the stitch density is the second most influential parameter on seam thread consumption because, in his case, the stitch density has a small interval (from 2 to 3.5 stitches/cm) [9]. Besides, Abhar calculated the contribution ratio percentage for stitch class 504 and concluded that the contribution ratio of stitch density was around $62 \%$ [11]. The stitch density has more impact because our studied stitches class 600 are made up of 4,5 , and 6 different threads. Each thread has its own path but the path of lopper thread is very curvy therefore, a little increase in stitch density contributes significantly toward the total thread consumption.

\subsubsection{Yarn diameter effect}

According to Figures $8 \mathrm{a}$ and $8 \mathrm{~b}$, Yarn linear density has little impact on thread consumption. But, the increase of yarn diameter from 0.024 to $0.036 \mathrm{~cm}$ (relative to increase of yarn linear density from 63.5 to 95 tex) increases thread consumption by $4.93 \%$ and $6.93 \%$ for 602 and 605 cover stitches, respectively. This result is similar to Jaouachi results [24]. In addition, if $100 \%$ PES thread composition is used instead of $100 \%$ cotton yarn, thread consumption becomes much less significant. In fact, the increase of its linear density does not have an important effect on thread consumption in the case of polyester thread composition.

\subsubsection{Material thickness effect}

Based on Figures $8 \mathrm{a}$ and $8 \mathrm{~b}$, it is found that that the increase of material thickness from 0.075 to $0.123 \mathrm{~mm}$ increases thread consumption by $3.05 \%$ and $4.02 \%$ for 602 and 605 cover stitches, respectively. Thus, material thickness does not have a very important effect, but it remains an influential factor in thread consumption. It is observed that the increase of material thickness increases thread consumption for the three studied cover stitches. It is noted that the increase of material thickness increases seam thickness as well as thread consumption. So it is deduced that less thick fabric requires less thread, but thicker fabric requires more thread. This result is confirmed by Jaouachi and Abher's finding that consumption values increase with the increase of material thickness $[1,6,11]$. This means that the thickness of fabric samples affects the consumed thread considerably. This finding is also in accordance with the findings of other researchers [24]. Besides, a heavy fabric consumes more sewing thread than an average fabric according to Jaouachi. This consumption is estimated for heavy fabrics that are thicker than the average fabrics [24]. However, Sharma proved that the material thickness has the most important influence on seam consumption because in his case, the material thickness has a very large interval (from1.64 to $6.56 \mathrm{~mm}$ ) [9].

\subsubsection{Seam width effect}

From the Figure $8 \mathrm{a}$ for 602-cover stitch, it is found that the seam width is an important parameter. Note that the increase in the seam width value $\left(B_{1}\right)$ increases the thread consumption. In fact, increasing $S_{W}$ from 0.3 to $0.6 \mathrm{~cm}$, thread consumption is increased by $30.29 \%$ for 602 -cover stitch. This is because the increase of width increases the stitch length. This result seems to be similar to the result found by Goldnfiber [27], who reported that higher width of seam requires more thread to sew the garments. 


\section{CONCLUSIONS}

This work deals with the prediction of the required amount of sewing thread using denim samples and cover stitch class 600 using more than three significant input parameters and analyzed their effects through two methods: mathematical (geometrical technique) and statistical (multi-linear regressive technique) methods. On comparing the results, it is found that the accuracy of the geometrical modeling method is not good to explain the consumption behavior in each cover stitch shape for class 600 .

Using statistical analysis, multi-linear regression models were obtained and investigated. Their corresponding $R^{2}$ values ranged from $97.05 \%$ to $99.63 \%$, illustrating their accuracy. Nevertheless, using geometrical modeling technique, the $R^{2}$ values ( $R^{2}$ ranged from $98.78 \%$ to $99.38 \%$ ) seem more significant than those given by the statistical method. This superiority encourages industrialists to apply the geometrical models for thread consumption prediction based on coverstitch geometry in class 600 .

Notwithstanding, to select the input parameters that are most influential on the consumption behavior, a classification of the significance using Minitab Software is applied. The increase of each parameter increases the seam thread consumption. The parameters that are most influential on the increase of thread consumption are the stitch density followed by seam width. The yarn diameter (relative to yarn linear density) and material thickness have little impact on thread consumptions values. Stitch density has more impact because stitches of class 600 are made by using 4, 5, or 6 different threads. Each thread has its own path but the path of lopper thread is very curvy, therefore, a little increase in stitch density contributes significantly to the total thread consumption.

We can conclude that the proposed models can accurately predict the thread consumption based on chain stitch class 600 because of the developed geometrical relationships. Therefore, it can be used to better estimate the required thread.

\section{References}

[1] Jaouachi, B., Aouine, S., Khedher, F. (2017). Consumed sewing thread behaviour based on Lockstitch and chain stitch. Indian Journal of Fibre \& Textile Research, 42(3), 325-334.

[2] Khedher, F., Jaouachi, B. (2014). Waste factor evaluation using theoretical and experimental jean pants consumptions. The Journal of the Textile Institute, 106(4), 402-408.

[3] Subrata and Vaseem. (2014)

[4] Lauriol, A. (1999). Modes and techniques: Initiation to the technology of materials in the clothing industries. (2nd ed.) Strasbourg, France.

[5] Midha, V. K., Sharma, S., Gupta, V. (2016). Predicting sewing thread consumption for lockstitch using regression model. Research Journal of Textile and Apparel, 20(3), 155-163.
[6] Abher, R., Sheraz, A., Muhammed, M., Faheem, A., Afzel, M. A. (2014). Geometrical model to calculate the consumption of sewing thread for 301 Lockstitch. The Journal of the Textile Institute, 105(12), 1259-1264.

[7] Jaouachi, B., Khedher, F. (2015). Evaluation of sewed thread consumption of jean trousers using neural network and regression methods. Fibres \& Textile in Eastern Europe, 3(111), 91-96.

[8] Jaouachi, B., Khedher, F. (2013). Evaluating sewing thread consumption of jean pants using fuzzy and regression methods. The Journal of the Textile Institute, 104(10), 1065-1070.

[9] Sharma, S., Gupta, V., Midha, V. K. (2017). Predicting sewing thread consumption for chain stitch using regression model. Journal of Textile Science and Engineering, 7, DOI: 10.4172/2165-8064.1000295.

[10] Vlinay, K. M., Shailja, S., Vaibhav, G. (2016). Predicting sewing thread consumption for lockstitch using regression model. Research Journal of Textile and Apparel, 20(3), 155-163.

[11] Abher, R., Sheraz, A., Ateequr, N., Muhammad, B. R. (2018). Geometrical model to calculate the consumption of sewing thread for 504 over-edge stitch. The Journal of the Textile Institute, 109(11), 1418-1423.

[12] Gazzah, M., Khedher, F., Jaouachi, B. (2017). Modelling the sewing thread consumption of 602 cover-stitch based on its geometrical shape. The International Journal of Applied Research on Textile, 5(2), 1-15.

[13] Chavan, Md.. V., Ghosh, S., Naidu, M. R. (2019). An elliptical model for lockstitch 301 seam to estimate thread consumption. The Journal of the Textile Institute, 110(12), 1740-1746.

[14] Jaouachi, B., Khedher, F., Adolphe, C. D. (2018). Compared basic stitch's consumptions using image analysis, geometrical modelling and statistical techniques. The Journal of the Textile Institute, 110(9), 1280-1292.

[15] Malek, S., Khedher, F., Adolphe, C. D., Jaouachi, B. (2019). Sewing thread consumption for chain stitches class 400 using geometrical and multi-linear regression models. Autex Research Journal, DOI: 10.2478/aut-2019-0051.

[16] Malek, S., Adolphe, C. D., Jaouachi, B. (2019). Prediction of sewing thread consumption for over-edge stitches class 500 using geometrical and multi-linear regression models. Autex Research Journal, DOI: 10.2478/aut-2019-0060.

[17] Malek et al. (2019).

[18] NFG 07-117. (1981). Method of assessing of fabric threads slippage and measurement of the seam resistance. French Association for Standardization AFNOR, 201-209.

[19] Mukhopadhyay, A. (2008). Relative performance of lockstitch and chainstitch at the seat seam of military trouser. Journal of Engineered Fibers and Fabrics, 3(1), 21-24.

[20] Jaouachi et al. (2019).

[21] Jaouachi et al. (2015).

[22] Sohanur. (2015).

[23] Gazzah, M. (2015). Study of the effect of yarn/yarn friction on the behavior bagging of denim fabric. PhD thesis in Textile Engineering, University of Monastir- Tunisia.

[24] Jaouachi, B., Khedher, F., Mili, F. (2012). Consumption of the sewing thread of jean pant using Taguchi design analysis. Autex Research Journal, 12 (4), 81-86. 
[25] Kennon, W. R., Hayes, S. G. (2000). The effects of feed retardation on chain stitch sewing. The Journal of the Textile Institute, 91(4), 509-522.

[26] Hayes, S. G. (2001). The Effects of check-spring travel on chain stitch sewing. Research Journal of Textile and Apparel, 5(2), 54-64.

[27] Goldnfiber. (2018). Major Factors for doing Sewing Thread Consumption. Web site: http://www.goldnfiber. com/2016/07/major-factors-for-doing-sewing-thread consumption.html

[28] Ghosh, S., Chavhan, Md. V. (2014). A geometrical model of stitch length for lockstitch seam. Indian Journal of Fibre \& Textile Research, 39(2), 153-156.
[29] Prabir, J. (2011). Assembling technologies for functional garments-An overview. Indian Journal of Fibre \& Textile Research, 36, 380-387.

[30] Sobuj, Md. S. R. (2015). Relation between yarn count and diameter \& count calculation for ply yarn. Web site: https:// textilestudycenter.com/relation-between-yarn-count-anddiameter (27 February 2015).

[31] Yildiz, Z., Dal, V., Ünal, M., Yildiz, K. (2013). Use of artificial neural networks for modelling of seam strength and elongation at break. Fibres \& Textiles in Eastern Europe, 21, 5(101), 117-123. 